\title{
COMMENTARY
}

\section{Platelets, endothelium and shear join forces to mislead neutrophils in sepsis}

\author{
Heitor A Paula Neto and Paul Kubes* \\ See related research by Ploppa et al., http://ccforum.com/content/14/6/R201
}

\begin{abstract}
Neutrophils are circulating leukocytes with great cytotoxic potential, responsible for the first combat against invading pathogens. Their accumulation in tissues must be highly controlled so that the number of neutrophils delivered to the affected site is sufficient to control infection with minimum injury to the surrounding healthy tissue. In sepsis, neutrophil migration is dysregulated - resulting in insufficient delivery of neutrophils to the infectious site and massive neutrophil accumulation in uninfected organs. This dysregulation has the potential to cause inappropriate tissue injury that may explain the multiple organ dysfunction observed in severe sepsis. A better understanding of the mechanisms that contribute to this process is fundamental to design therapeutic strategies to circumvent tissue injury and organ dysfunction in sepsis.
\end{abstract}

In the previous issue of Critical Care, the report by Ploppa and colleagues reveals an interrelation among neutrophil activation, endothelial cells, platelets and shear forces that may underlie the unwanted accumulation of neutrophils in vascular beds unrelated to the infection site [1].

A hallmark feature of neutrophils is their ability to rapidly accumulate at sites of infection, where they kill invading pathogens through the release of their cytotoxic granule contents, the generation of reactive oxygen species and, under certain critical conditions, the release of enzyme-laden DNA that forms large web-like structures or neutrophil extracellular traps [2]. The movement of neutrophils out of the vasculature into

\footnotetext{
*Correspondence: pkubes@ucalgary.ca

Immunology Research Group, Department of Physiology and Pharmacology, Calvin, Phoebe \& Joan Snyder Institute for Infection, Immunity \& Inflammation, University of Calgary, 3330 Hospital Drive NW, Calgary, AB, Canada T2N 4N1
}

tissues follows a well-described process that involves their weak interaction with the endothelium through selectins and subsequent firm adhesion mediated by integrins [3]. This migration process must be highly controlled, since insufficient numbers of neutrophils in the infectious site results in a failure to control infection, whereas excess neutrophil accumulation may lead to tissue injury.

Studies carried out over the past decade showed that, during sepsis, there is an important dysregulation in neutrophil migration. This is best characterized by an insufficient recruitment of neutrophils to the primary site of infection along with a massive accumulation of neutrophils in distal organs such as the lungs and liver. The clinical relevance of these phenomena is the strong relationship between neutrophils in organs and tissue injury leading to subsequent multiple organ dysfunction, the ultimate cause of death in sepsis $[4,5]$.

The study by Ploppa and colleagues used a simplified in vitro flow chamber model and revealed the paradoxical finding that activated neutrophils adhere less well than unstimulated neutrophils to activated endothelium, in part due to shedding of the adhesion molecule L-selectin [1]. Second, these activated neutrophils only adhered firmly to the endothelium when shear was significantly decreased. Third, mimicking the endothelial injury (presumed to occur in sepsis) by exposing the subendothelial matrix led to platelet adhesion that mediated a profound increase in subsequent adherence of activated neutrophils even in the presence of higher shear forces.

The authors then attempted to translate these results to neutrophil behavior in septic patients. The authors postulated that neutrophils, upon activation, would preferentially accumulate in vascular beds with poor perfusion. There is a huge body of work showing that circulating neutrophils are activated by a myriad of proinflammatory mediators during sepsis [6,7]. Whether the disproportionate neutrophil recruitment into, for example, the lungs is due to poor perfusion, whether it is due to the unique geometry of the pulmonary microvasculature or whether it is due to unique adhesive profiles found on lung endothelium, however, remains unclear. Certainly, 
the limitations of this study include the inability to mimic the geometry of pulmonary capillaries and the use of human umbilical vein endothelium, which differs in a major way from pulmonary endothelium [8]. The authors also postulate that the accumulation of activated neutrophils in vascular beds could trigger massive endothelial damage, exposing the subendothelial matrix to circulating platelets. Consequently, platelets would aggregate at these sites and could mediate further neutrophil accumulation less dependent on shear and more dependent on P-selectin expressed by the platelets. Together, the poorly perfused beds could become sites of preferentially activated neutrophil trapping leading to increased endothelial injury, which would be followed by platelet deposition and further neutrophil accumulation, inducing a vicious cycle that would ultimately lead to loss of organ function.

A strength of this study is the use of human cells, which is not trivial as significant differences exist between humans and mice - even for example in the synthesis of P-selectin, which can occur via NF- $\mathrm{kB}$ in rodents but requires completely different cytokines (for example, IL-4) in humans [3]. Mouse experiments can, however, provide important information. For example, neutrophils arrive prior to platelets in some endotoxemic organs, making neutrophil sequestration independent of platelets [9]. Moreover, platelets will bind already adherent neutrophils in the septic milieu and will activate and induce neutrophil extracellular traps, and this does cause massive endothelial injury, highlighting the further complexity of the in vivo septic milieu [10]. Moreover it was recently demonstrated that neutrophils in a septic milieu express novel chemokine responsiveness [7] and novel adhesion pathways turning off integrins via IL-10 and activating novel adhesive mechanisms such as CD44/ hyaluronan in places such as the liver $[11,12]$. This begs the question of whether adhesion is different in each and every organ in sepsis [3], making anti-adhesion therapy a less attractive approach.

The report by Ploppa and colleagues reaffirms the general view that even in a simple three-cell in vitro system under flow conditions there can be significant complexity underlying neutrophil trafficking. However, it is provocative to question whether this is truly dysregulation or a final attempt by the immune system to squelch a systemic infection by setting up neutrophil-platelet road blocks in various vasculatures in an attempt to catch circulating bacteria. Indeed, neutrophil-platelet interactions in sepsis cause the release of neutrophil extracellular traps that trap bacteria in a very effective manner [10]. Clearly, effort is needed to move from these in vitro systems to imaging the human vasculature to gain further understanding of this process in order to translate data into increased patient survival in intensive care units.

\section{Abbreviations}

IL, interleukin; NF, nuclear factor.

\section{Competing interests}

The authors declare that they have no competing interests.

\section{Acknowledgements}

HAPN receives a postdoctoral fellowship from Coordenação de

Aperfeiçoamento de Pessoal de Nível Superior (CAPES, Ministério da Educação, Brasil).

Published: 14 January 2011

\section{References}

1. Ploppa A, Schmidt V, Hientz A, Reutershan J, Haeberle HA, Nohé B: Mechanisms of leukocyte distribution during sepsis: an experimental study on the interdependence of cell activation, shear stress and endothelial injury. Crit Care 2010, 14:R201.

2. Borregaard N: Neutrophils, from marrow to microbes. Immunity 2010, 33:657-670.

3. Petri B, Phillipson M, Kubes P: The physiology of leukocyte recruitment: an in vivo perspective. J Immunol 2008, 180:6439-6446.

4. Alves-Filho JC, Spiller F, Cunha FQ: Neutrophil paralysis in sepsis. Shock 2010, 34:15-21.

5. Reddy RC, Standiford TJ: Effects of sepsis on neutrophil chemotaxis. Curr Opin Hematol 2010, 17:18-24.

6. Muller Kobold AC, Tulleken JE, Zijlstra JG, Sluiter W, Hermans J, Kallenberg CG, Tervaert JW: Leukocyte activation in sepsis: correlations with disease state and mortality. Intensive Care Med 2000, 26:883-892.

7. Souto FO, Alves-Filho JC, Turato WM, Auxiliadora-Martins M, Basile-Filho A, Cunha FQ: Essential role of CCR2 in neutrophil tissue infiltration and multiple organ dysfunction in sepsis. Am J Respir Crit Care Med 2010, in press. doi:10.1164/rccm.201003-04160C.

8. Hillyer P, Mordelet E, Flynn G, Male D: Chemokines, chemokine receptors and adhesion molecules on different human endothelia: discriminating the tissue-specific functions that affect leucocyte migration. Clin Exp Immunol 2003, 134:431-441.

9. Andonegui G, Kerfoot SM, McNagny K, Ebbert KV, Patel KD, Kubes P: Platelets express functional Toll-like receptor-4. Blood 2005, 106:2417-2423.

10. Clark SR, Ma AC, Tavener SA, McDonald B, Goodarzi Z, Kelly MM, Patel KD, Chakrabarti S, McAvoy E, Sinclair GD, Keys EM, Allen-Vercoe E, Devinney R, Doig CJ, Green FH, Kubes P: Platelet TLR4 activates neutrophil extracellular traps to ensnare bacteria in septic blood. Nat Med 2007, 13:463-469.

11. McDonald B, McAvoy EF, Lam F, Gill V, de la Motte C, Savani RC, Kubes P: Interaction of CD44 and hyaluronan is the dominant mechanism for neutrophil sequestration in inflamed liver sinusoids. J Exp Med 2008, 205:915-927.

12. Menezes GB, Lee WY, Zhou H, Waterhouse CC, Cara DC, Kubes P: Selective down-regulation of neutrophil Mac-1 in endotoxemic hepatic microcirculation via IL-10. J Immunol 2009, 183:7557-7568.

doi:10.1186/cc9371

Cite this article as: Neto HAP, Kubes P: Platelets, endothelium and shear join forces to mislead neutrophils in sepsis. Critical Care 2011, 15:103. 